\title{
A Measurement Instrument for Enterprise Architecture Resilience Research: A Pilot Study on Digital Transformation
}

\author{
Adina Aldea \\ University of Twente \\ a.i.aldea@utwente.nl
}

\author{
Amitrajit Sarkar \\ Ara Institute of Canterbury \\ Amit.Sarkar@ara.ac.nz
}

\begin{abstract}
Enterprise Architecture can contribute positively to enterprise resilience. In this connected dynamic world Enterprise Architecture Resilience is an emergent area of Information Systems research that is characterized through an abundance of conceptual work with little empirical research. To fill this gap, this paper reports on the development and validation of an instrument to measure various resilience attributes and barriers in context to digital transformation in organizations. We advance an extended model for a multi-stage measurement instrument development procedure, which incorporates feedback from both academics and practitioners. We identify two main contributions: First, we provide a validated measurement instrument for the study of Enterprise Architecture Resilience factors in context to digital transformation in organizations, which can be used to assist in further empirical studies that investigate phenomena associated with the enterprise architecture domain. Second, in doing so, we describe in detail a procedural model for developing measurement instruments that ensure high levels of reliability and validity, which may assist fellow scholars in executing their empirical research.
\end{abstract}

\section{Introduction}

In uncertain, turbulent, dynamic, continuously evolving environments only adaptable, agile, and relentlessly dynamic organizations will survive and forge ahead. In this digital era, organizations are increasingly dependent on Information Systems (IS) for both innovation and operations. Organizations of any type and size increasingly rely on complex IS and digital platforms to manage their businesses, which require IS to operate dependably under a range of adverse circumstances. Previous research has addressed business continuity planning, contingency planning, emergency planning, disaster recovery, and other pertinent issues. Organizational research has included all of these issues in the concept of organizational resilience, which is commonly defined as the organization's ability to operate reliably under a range of adverse circumstances, but the concept of IS resilience has yet to be developed [1].

The resource-based view of firms describes a firm as a specific collection of resources and capabilities that can be deployed to achieve a competitive advantage. Firm resources are defined as all tangible and intangible assets and capabilities belonging to or controlled by a firm that can be used to implement competitive strategies [2]. Firm-specific resources and capabilities must be protected and made difficult to transfer, imitate, or replicate [3]. Therefore, by protecting their valuable resources against imitation or substitution, firms can sustain existing advantages. RBV in dynamic environments where rapid and sudden changes are common. To address this issue, the dynamic capability emerged to explain how firms react, adapt, and respond to changes in volatile environments. The dynamic capability perspective suggests that new forms of competitive advantage are achieved by creating new resources to achieve congruence with the changing environment and emphasizes understanding how organizations develop new resources and capabilities to support business strategy in rapidly changing environments [2].

In recent times, the COVID-19 pandemic has been spreading globally and has affected numerous countries, societies, and organizations. As of April 2021, the virus had already caused more than 2.5 million deaths worldwide. However, the impact of the pandemic extended beyond the health status of the population and damaged economic development as well. The effects of the pandemic ranged from delivery bottlenecks by suppliers to the partial stoppage of the company's production facilities. To counteract such risks, research in the areas of disaster preparedness and recovery, as well as business continuity management, has been conducted for years. The findings from these areas are intended to provide solution approaches with which a company can prepare itself for crises and, if the scenario occurs, can survive them. 
One of the aspects that the COVID-19 pandemic has highlighted over the past few months, is that there is a strong need for enterprise-wide resilience. With the awareness and better understanding of cloud platforms, it is forecasted that implementation of cloud solutions will increase significantly, and the next wave of digital transformation is at doorstep. This transformation can be designed using Enterprise Architecture (EA). Specifically, the ability of organizations to design resilient EAs and to assess EA resilience is becoming increasingly critical for dealing with uncertainty. However, even though the concept of resilience is widely studied since the seventies, little research and literature is available about EA resilience and its implications; little is known about the possible approaches that organizations and EA practitioners can consider to EA resilience and its related design, measurement, and control. In turn, there is a need to have a better understanding of published research on EA resilience to incentivize further discussion. Also, it is critical to identify the success factors and the barriers of EA resilience and to create an instrument that can measure the level and maturity of EA resilience in context to the digital transformation in organizations. This research paper responds to this need. To our best knowledge, at this point in time, no instrument has been developed nor empirically tested relating to EA resilience [4].

The goal of this paper is to explore and potentially overcome the identified research gap in Enterprise Architecture (EA) literature regarding Resilience preparedness in the case of Digital transformations. Thus, to help achieve this goal, we have formulated the following research question:

Which factors are relevant for Enterprise Architecture Resilience preparedness for Digital transformation in organizations?

By answering this research question, we aim to provide useful insights for decision-making to both EA practitioners and the top management team within an organization. More specifically, in this paper, we aim to design an instrument to:

- Provide an indication of resilience priorities for Enterprise Architecture design.

- $\quad$ Provide a validated set of generic EA Resilience factors for Digital transformations.

- Confirm the extent to which EA Resilience preparedness for Digital transformation is considered necessary.

This paper is structured as follows. Section 2 presents the theoretical framework and conceptual that is used as a basis for designing our survey questionnaire instrument, which is described in Section 3. In Section
4, we introduce the results of our pilot study and in explain how this has led to changes in the questionnaire. The paper concludes in Section 5 with a discussion on the contributions of our work, the limitations and future work.

\section{Theoretical framework 2.1. IT Governance}

IT governance, the term defined as "specifying the decision rights and accountability framework to encourage desirable behavior in the use of IT" [5], constitutes the most universal and systematic approach to solve the problems connected with supporting the business with IT in the organizational context. According to Weill and Ross [5], IT governance encompasses five major decision domains:

- IT principles comprise the high-level decisions about the strategic role of IT in the business.

- IT architecture includes an integrated set of technical choices to guide the organization in satisfying business needs.

- IT infrastructure consists of the centrally coordinated, shared IT services that provide the foundation for the enterprise's IT capability and were typically created before precise usage needs were known.

- Business application needs are the business requirements for purchased or internally developed IT applications.

- Prioritization and investment decisions determine how much and where to invest in IT.

When defining these five decision domains for IT, Weill and Ross [5] are mostly focusing on aspects of Information Systems (IS) and how they relate to the business aspects of an organization.

When relating these five decision domains to the topic of our research, we can argue that all of them are covered to some degree. First, resilience can be considered a principle that guides the decisions of an organization. Enterprise resilience can help organizations with managing unexpected events by identifying vulnerabilities, having the ability to change and adapt, and recovering quickly from unexpected events [6]. Second, EA is a discipline that focuses on the holistic management of the enterprise, based on the elements from its architecture (e.g.: business processes, applications, information, hardware, etc.) as well as the relations between them [7]. Thus, it covers the management, design and implementation of and architecture and its corresponding elements. Furthermore, EA is identified to improve the Business and IT alignment in an organization [8], which implies that business needs are considered when designing the 
EA. Finally, in the past few decades, many of the investment organizations have made can be related to their Digital transformation initiatives [9]. This effect has also been amplified due to the COVID-19 pandemic, where many organizations had to invest in and implement digital solutions to deal with the impact of the pandemic [10]. Thus, Digital transformation can be seen as one of the drivers for investment in IT.

Another description by De Haes and Van Gembergen [11] describes IT Governance as "the organizational capacity ... to control the formulation and implementation of IT strategy". Executed by various administrative bodies, the aim is to merge the demands and objectives of the business with those of the IT unit. This interpretation overlaps with the following definition describing IT Governance as the interplay of management, organizational structures, and processes through which business intentions can be carried out without simultaneously neglecting IT [11]. This study takes the essence of both definitions and emphasizes the "alignment of business and IT, and the primary responsibility of the board and senior management.

IT governance can be deployed using a mixture of various structures, processes, and relational mechanisms.IT governance structures include organizational units and their roles and responsibilities for making IT decisions. An example of these structures, processes and relational mechanisms are provided in Figure 1.

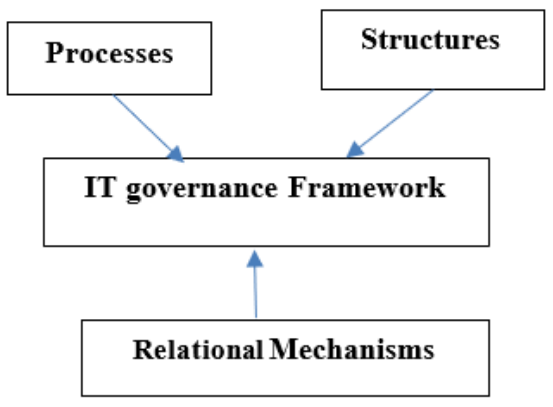

Figure 1. IT Governance Framework

In this research the focus is on to develop an instrument to identify who, what and how decisions are prioritized to ensure EA resilience, this will be an important contribution of this research.

\subsection{Resilience}

The concept of resilience has been a prominent and emerging topic in various scientific fields. However, as resilience research encompasses a wide range of disciplines (e.g.: ecology, psychology, engineering, organization management, etc.), as well as different research contexts and topics, it is not surprising that the concept lacks an accepted common definition across disciplines [12].

The first definition of resilience was introduced by Holling [13] in his work on stability and resilience in ecological systems. Here, resilience is defined as the capacity of a system to adapt to change and deal with surprise while retaining the system's basic function [13].

The earliest research on organizational resilience describes resilience as a purely defensive reaction, while a change of understanding has taken place, resulting in concentrating on adaptation and anticipation [14-16].

The aspect of moving forward and expanding business actions during uncertain events also distinguishes a resilient firm from a robust organization [16]. However, resilience and robustness are intermediate stages on the way to develop from a fragile organization to an antifragile, with antifragile enveloping the state of resilience and the skill to prosper during disturbances [17].

Scholars describe the coupling of capabilities, resources, characteristics, and abilities of an organization [17] as key steps for becoming resilient. Duchek [16] divides organizational resilience into the three stages of anticipation, coping, and adoption, for the time intervals before, during, and after an event. The importance of capabilities and their anchoring in the social context is emphasized as the main drivers during the coping stage.

Denyer [15] describes the level of resilience as mainly influenced by the behaviors and actions during the stages of preventative control, mindful action, performance optimization, and adaptive innovation. For mature resilience, one must balance resources, objectives, actions, and desires among these stages.

For our research, we focus on defining and understanding resilience from the perspective of two interrelated domains. Namely, IS resilience, which has been studied for the past decade, and EA resilience which has only recently been investigated by academics.

\subsection{IS Resilience}

According to Sarkar et al. [18], a clear interpretation of IS resilience was nonexistent at the time of their research. Therefore, they defined IS resilience as follows:

IS resilience is a function of an organization's overall situation awareness related to Information Systems, management of Information Systems vulnerabilities, and adaptive capacity, risk intelligence, flexibility, and agility of Information Systems in a complex, dynamic, and interconnected environment.

According to this definition, IS resilience is based on six attributes - situation awareness, management of 
vulnerabilities, adaptive capacity, risk intelligence, flexibility, and agility [18]. The attributes adaptive capacity, risk intelligence, flexibility, and agility suggest that IS resilience also addresses initiative-taking measures to adapt and improve IS. Therefore, the robustness of the system is not the sole focus point, but an intermediate. This proactive tone also characterizes organizational resilience, which shows that IS resilience is a subarea of organizational resilience. These terms are defined in Table 1.

Another perspective on IS resilience is provided by Aldea et al. [4]. The authors perform a systematic literature review where they investigate available research to determine the state-of-the-art on several aspects, including the characteristics of IS resilience and the strategies for IS resilience.

Aldea et al. [4] present a list of IS resilience characteristics gathered from multiple papers that expands on the attributes provided by Sarkar et al. [18]. Namely, the following characteristics are mentioned: diversity, efficiency, adaptability, cohesion, selforganization, robustness, learning, redundancy, rapidity, flexibility, equality, agility, vulnerability to risk, responsiveness.

Following the classification by Ramezani and Camarinha-Matos [19], the authors identify 33 resilience strategies that organizations can use. The strategies are classified based on three phases of resilience, namely Readiness (preparation for disturbances), Responsiveness (once the disruption has occurred), Recovery (after the disruption has ended). Some of the strategies mentioned range from creating a disruption management culture, forecasting and risk assessment (Readiness), to acceptance of the consequences, revision of the systems and postponement of decision-making (Responsiveness), to collaboration with all stakeholders, knowledge management and sense-making (Recovery).

\subsection{EA Resilience}

To the best of our knowledge, Aldea et al. [4] were the first to coin the term EA resilience and to provide a definition for it, namely:

EA resilience is the ability of an organization to identify and assess the vulnerabilities of enterprise resources in its integrated architecture and prepare for disruptions, by designing specific measures in an EA to increase its capabilities to adapt to new or changing circumstances and restore full capability after an unexpected disruption.

Bemthuis et al. [20], present another view on EA resilience by studying it from the perspective of cyberphysical systems of systems and emergent behaviors. Additionally, the authors coin the term Enterprise resilience as a type of resilience that can be facilitated by designing EA models.

The idea of resilience by design is relatively new and it originates from the field of civil engineering. After the disaster brought on by Hurricane Sandy, the US Department of Housing and Urban Development proposed a new way to address the structural and environmental vulnerabilities exposed by the hurricane and to develop solutions that could provide better protection from natural events [21]. Thus, according to Linkov et al. [22], the resilience by design paradigm defines that a system must be designed to recover its functions from disruption on its own or else the system will fail. In terms of EA, this would translate to the idea that the EA of an organization must be designed with resilience in mind.

Regarding the attributes and strategies for EA resilience, Aldea et al. [4] argue that all of the ones identified for IS resilience might be suitable for EA as well. However, the authors only theorize this and do not provide any validation of their assumption. In our current paper, we aim to address this issue by designing a survey questionnaire that includes several items regarding the suitability of these attributes and strategies for EA resilience.

To study the value-generating mechanisms of EA resilience, we use the resource-based view (RBV) as a theoretical lens. The RBV has been applied successfully to explain how value is generated through the use and management of IT. In this study, we opt for an in-depth literature review, which extends the systematic literature review by Aldea et al. [4], to develop an instrument to investigate the resources and capabilities developed with EA Governance and analyze how they improve existing IS capabilities.

It will be most useful for the present investigation to focus on the basic kinds of resources that are most relevant in the context of EA Resilience: (1) human beings taking over EA roles, (2) the technological assets used for documenting and analyzing a firm's EA, and (3) the standards, techniques, and process models, etc. guiding a firm's EA Governance[23].

\subsection{EA Maturity}

Maturity models are typically used to measure and assess the current level of competence of an organization in a particular domain [24]. One of the earliest maturity models that have served as a basis for many of the models in the software and IT domain is the Capability Maturity Model.

According to Vallerand et al. [25], several other maturity models have been developed over the years, such as the CMMI, Gartner's IT Score for EA, Forrester's EA maturity assessment tool, the Center for 
Information Systems Research at MIT EA management maturity framework, etc.

Meyer et al. [24] provide a comparison of several maturity models that were available at the time, such as IT-CMF, EAMM, SAMM, COBIT, and more. The authors conclude that the IT-CMF is the most suitable one to assess the EA maturity of an organization due to its ability to provide both a high-level and in-depth analysis.

Another maturity model that is identified by both Meyer et al. [24] and Vallerand et al. [25] is the Architecture Capability Maturity Model (ACMM) developed by the US Department of Commerce. The ACMM is notable because it has been adopted and incorporated in the TOGAF standard [26], which is the most used EA framework by practitioners [27].

Finally, a recent trend in EA research has emerged which identifies EA agility as a means to address EA complexity and the dynamic environment of organizations [28]. In line with this, the Open Group has started developing the Open Group Agile Architecture Framework which contains an Agile Architecture Maturity Model (OAMM) that extends the CMMI with agile concepts [29].

For our research, we have considered including the IT-CMF, the ACMM and the OAMM. However, the ITCMF is fairly detailed due to the 37 capabilities that need to be assessed. Thus, we considered it not suitable for inclusion in our survey questionnaire since EA maturity is just one aspect of our research and not its main focus. On the other hand, the ACMM and the OAMM are relatively high-level and cover two important aspects that we would like to include. First, the ACMM can help assess the EA maturity of organizations utilizing the traditional measures and understanding of the EA practice. Second, the OAMM can be used to assess the degree to which agility is incorporated in the EA practice. Considering that agility is one attribute of EA resilience, including the OAMM, can help with understanding the relationship between EA maturity and resilience.

\subsection{Digital Transformation Maturity}

The COVID-19 pandemic has led to a lot of organizations undertaking digital transformation programs to be able to operate properly in this disruptive period [10]. However, digital transformation is not a new trend. It has been pursued by many organizations to a varying degree of success since the diffusion of the Internet at the turn of the millennium [30].

Digital transformation can be seen as an ongoing process of adopting digital technologies to meet the expectations of customers, employees and partners [31]. It is seen as a way for organizations to transform their business models quickly by implementing new digital capabilities based on technologies, such as $5 \mathrm{G}$, artificial intelligence, 3D printing, social media, cloud computing, big data and analytics, etc. $[10,31]$. Thus, there is a natural relation between digital transformation and EA. Anthony Jnr [32] argues that EA facilitates digital transformation by enabling an alignment between digital technologies and business models, and by increasing the success of the transformation.

However, digital transformation is not only about implementing new digital technologies. It facilitates a complete change of an organization from multiple perspectives, such as vision, strategy, organizational structure, processes, capabilities, culture, etc. [9]. Thus, several stakeholders need to be involved in prioritizing investments and managing this transformation.

For this purpose, several digital maturity models to help assess the state of the organization's digital initiatives have been formulated over the years. Teichert [31], performed a systematic literature review that analyzed several maturity models both from academia and practice and identified the maturity areas that are assessed by them. These areas cover the following aspects: digital culture, technology, operations and processes, digital strategy, organization, digital skills, innovation etc. [31].

Similarly, to EA maturity models, these digital transformation areas (also called dimensions) are assessed with the help of certain maturity levels. Valdez-de-Leon [33] provides an example of these maturity levels: level 0 (not started), level 1 (initiating), level 2 (enabling), level 3 (integrating), level 4 (optimizing), and level 5 (pioneering). When comparing these levels to the OAMM, we can see that there are quite some similarities, even though the naming differs.

Rossmann [34] provides another perspective on digital transformation maturity assessment. Instead of relying on the maturity levels and the high-level dimensions, the author specifies for each dimension four items. These items are statements that can be assessed based on a Likert scale ranging from strongly disagree to strongly agree.

For our research, we choose to use a combination of the aforementioned research papers. We choose to use a combination of the dimensions proposed by Teichert [31] and Rossmann [34]. Furthermore, we incorporate the maturity levels as defined by Valdez-de-Leon [33].

\subsection{Conceptual Model of EA Resilience in the context of Digital Transformation}

Based on the literature from Sections $2.1-2.6$, we have formulated a conceptual model to structure our research. In this model we have included three core concepts, namely: 
- $\quad$ EA maturity as the level of competence that an organization has in the EA practice.

- Digital transformation maturity as the level of the organization's digital initiatives.

- EA resilience as the ability of an organization to design their EA to prepare for disruptions.

From the reviewed literature, several relations between these three concepts can be concluded:

- Organizations that have an EA practice implemented can lead to successful digital transformation.

- Organizations that have an EA practice can design their EA with resilience in mind.

- EA resilience can have several characteristics/attributes and certain resilience strategies can be used to address disruptions.

Based on this information, we formulated the conceptual model which can be seen in Figure 2.

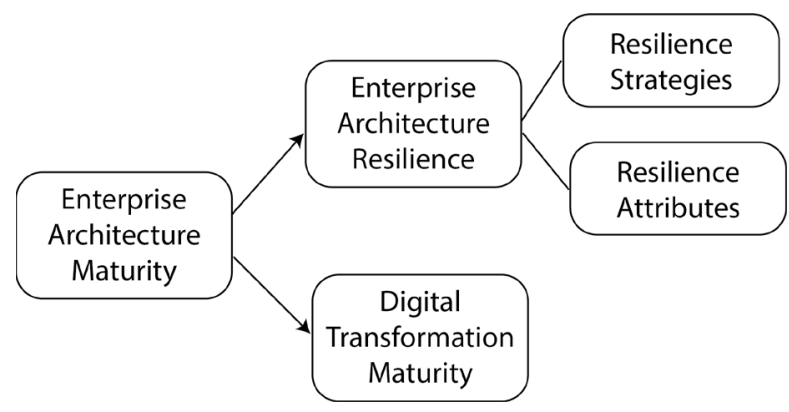

Figure 2. Conceptual model

The conceptual model has led to the formulation of three hypotheses which will be tested with the help of the survey questionnaire instrument that we present in Section 3. We have chosen to refine the concept of EA resilience into Resilience Strategies and Resilience Attributes. Thus, we consider that an organization that aims at incorporating resilience in their EA should consider implementing several resilience strategies and attributes.

H1: Enterprise Architecture maturity positively influences the Digital Transformation maturity of the organization.

H2a: Enterprise Architecture maturity positively influences the number of Resilience Strategies that are being implemented by the organization.

H2b: Enterprise Architecture maturity positively influences the number of Resilience Attributes that are being considered by the organization.

\section{Survey Questionnaire Instrument Design}

\footnotetext{
${ }^{1}$ https://bit.ly/3vo6zPz
}

Quantitative data collection methods can provide a generalizable understanding of a topic [35]. One of the most used quantitative data collection methods is the survey questionnaire. Due to its nature, it is very suitable for collecting large amounts of responses in a less timeconsuming manner [36].

One of the main aspects to pay attention to with questionnaires is sample bias which can lead to concerns regarding the generalizability of the results [36]. This can be addressed by ensuring a large number of responses. For our research, we aim to distribute the questionnaire through many different local and international channels to ensure the generalizability of our results.

Quantitative methods such as questionnaires are usually criticized due to their limitation on understanding the context in which the respondents are expressing their opinions [35]. Thus, a mixed-methods approach combining quantitative and qualitative research can help ameliorate this. To address this in our research, we will ask the respondents to indicate whether they would be willing to participate in a followup interview to dive deeper into the topic of EA resilience in the context of digital transformation.

According to Lietz [37], there are several best practices when formulating questions and answers for questionnaires. For questions, the length should be a short as possible while still including all necessary information, grammar complexity should be kept to a minimum by using an active voice, negatively worded questions should be avoided, and the order of questions should be from the most generic to the most specific. Regarding the answers, there are a lot of best practices regarding the usage of scales, adding a don't know option, and the order of the answers. However, there are no straightforward recommendations for the answers, as it depends on what the goal of the questionnaire is.

The questionnaire has been created with the Qualtrics tool and has been published online ${ }^{1}$. In the following sections, the design of the questionnaire is explained together with the relation between the questions and the literature that supports them.

\subsection{Starting Page and Consent Form}

Since this is a self-administered questionnaire, the starting page of the questionnaire needs to provide enough information about the research that is performed for the respondent to make an informed decision about their participation. Thus, several aspects are included. Namely, the purpose of the research, what is expected from the respondent if they choose to participate, how the data collected will be managed and processed 
according to the General Data Protection Regulation of the EU, how the results of the research will be used, and how the respondents can contact the researchers.

The consent form includes a series of statements that describe what the participants agree to if they choose to participate in the research. Some examples are that they understand what is required and agree to participate in the research, that they understand that the opinions they provide will be kept confidential and will not identify the respondents or their organization, that they can contact the researchers if they have questions, etc. The respondents can start the questionnaire only if they give their explicit consent by ticking a checkbox and submitting their response.

\subsection{Background Information}

To have a better understanding of the context of the respondents, several questions are formulated. These questions cover aspects such as the industry their organization operates in, the size of the organization, the geographical location of their office, the role of the respondents within the organization, and their main responsibilities.

This type of information can help with identifying if there are certain characteristics of the organizations or the respondents which can justify the results obtained in the other sections of the questionnaire.

\subsection{Enterprise Architecture Maturity}

As indicated in the conceptual model from Figure 1, EA maturity is one of the core concepts that we would like to investigate. For this purpose, we have chosen to include several questions on aspects, such as the current maturity of the EA practice and the types of EA frameworks and modelling languages that are used by the organization.

Thus, this allows us to analyze whether organizations that have different levels of EA maturity also exhibit differences in the other areas from the conceptual model. Furthermore, we can relate the usage of certain frameworks and modelling languages to the maturity of the EA practice to get a more in-depth understanding.

\subsection{Digital Transformation Maturity}

Similarly, to the EA maturity section of the questionnaire, we have also defined several questions on Digital transformation maturity. These questions include an assessment of the current digital transformation initiatives of the organization on several dimensions and an evaluation of the impact and struggles due to COVID-19 from a digital transformation perspective.
While it would have been sufficient to ask respondents to assess their Digital transformation maturity, we consider that it is important to also understand the changes brought on by the pandemic. This information can be used to explain certain outliers as many organizations had to implement Digital transformation initiatives very quickly to mitigate the impact of the pandemic on their operations.

\subsection{EA Resilience Strategies and Attributes}

Two similar sections of the questionnaire are dedicated to understanding several aspects regarding EA Resilience. In both sections, respondents are provided with a list of Resilience Strategies and a list of Attributes, respectively. The purpose of this is to understand which Resilience Strategies and Attributes are currently implemented by the organization of the respondent and which ones they consider to be relevant to EA resilience.

Additionally, there are a few other questions relating to the support from top management for implementing EA Resilience, best practices to ensure EA Resilience, and how Resilience by design could be implemented in an EA practice.

Thus, these questions provide an insight into the status of EA resilience with the organizations of the respondents and give an overview of which aspects are considered by the respondents are important for ensuring EA resilience.

\subsection{General Feedback and Follow-up}

In the concluding section of the questionnaire, we ask the participants whether they would like to provide any additional feedback. The purpose of this is to gather any information that was not covered by the other sections of the questionnaire.

Additionally, the respondents are asked whether they would like to participate in a follow-up interview and/or if they would like to receive the results of this research. For both questions, if the respondents agree, they are asked to fill in their email address as a means for the researchers to contact them.

\section{Pilot Study and Instrument Verification}

For our survey questionnaire instrument, a set of 25 questions, was developed according to the guidelines delineated by earlier research [38]. In this section, we describe a procedural model for developing valid and reliable measurement instruments for theoretical constructs. This procedural model is proposed for use by researchers who wish to create new measurement 
instruments for conceptually defined theory constructs. The procedural model is not concerned with developing theory, instead, it applies to the stage of the research where such theory exists and is sought to be empirically tested.

The first stage of the procedural model is item creation, which is concerned with specifying the theoretical constructs for which measurement items are to be developed and deriving pools of candidate items for each construct. This task is carried out through an analysis of the relevant literature. The next stage is substrata identification, the purpose of which is to sort the candidate items into meaningful separate domain subcategories to display construct, convergent, and discriminant validity. This task is carried out with the help of a panel study with experts of the selected domain of study, which provides input to the sorting task. The third stage is item identification, the purpose of which is to identify from the pool of candidate items a revised set of items that show good potential for high content validity. This task is also carried out employing an expert panel study, which provides input to the ranking task. The fourth stage is item revision, the purpose of which is to re-specify and further improve the set of candidate items as well as to get an initial indication of reliability and validity. This task is carried out through a practitioner and expert panel study, to obtain input from a sample representative of the target research population. The last stage is instrument validation, which is concerned with obtaining statistical evidence for the reliability and validity of the developed measurement items. This task is carried out using the survey research method to obtain a sufficiently large number of responses from the target population of the respective study [38].

A set of statements should represent the concourse of interest in the same way that a sample of people should represent the population in a classical correlational study. Therefore, certain prescribed guidelines are adopted in the selection of statements to achieve the highest probability of "representativeness" of the domain of the concourse: 1) review of the literature, 2) conversations and interviews with people who participate in the concourse, and 3) input from domain experts about the content of the sample of statements.

A pilot test was conducted on a small convenience sample of IT professionals from different organizations in New Zealand and The Netherlands to obtain feedback from respondents about the length, content, and structure of the questionnaire. For this pilot study, we have used a convenience sample of Senior Practitioners to help as evaluators with the item selection phase. For this research, a CIO $(n=1)$ of a large organization, several IS professionals $(n=4)$ and academics $(n=3)$, and domain experts $(n=2)$ were recruited to help as evaluators with the item selection phase. Following the guidelines for instrument development, feedback from the evaluators was incorporated into the statement and EA Resilience instrumentation. After several iterations of the instrument development guidelines, the evaluators confirmed that the instrument is ready and should function as intended. We then approached all participants from New Zealand and the Netherlands, to answer the questionnaire to test the statistical properties of the question set and evaluate the survey instrument. Furthermore, to test the theoretical structure incorporated into the survey question set, a CIO of a large public organization was recruited to provide his/her suggestions. Respondents spent 20 to 30 minutes completing the questionnaires.

We used a semi-structured interview template of topics to cover. We specifically asked for, (a) the length of the questionnaire, (b) is there any questions missing, (c) The clarity of each question along with the category that they are placed in, (d) whether any question has been included which should not be added, (e) whether the same question has been added more than once.

The kappa statistic is frequently used to test interrater reliability. The importance of rater reliability lies in the fact that it represents the extent to which the data collected in the study are correct representations of the variables measured. Measurement of the extent to which data collectors (raters) assign the same score to the same variable is called interrater reliability. Cohen's kappa was developed to account for the possibility that raters guess on at least some variables due to uncertainty. Like most correlation statistics, the kappa can range from -1 to +1 .

All the kappa coefficients were evaluated using the guideline outlined by Landis and Koch [39], where the strength of the kappa coefficients $=0.01-0.20$ slight; 0.21-0.40 fair; 0.41-0.60 moderate; 0.61-0.80 substantial; 0.81-1.00 almost perfect. Overall percent agreement was $98 \%$ and kappa $=0.92$, which signifies almost perfect agreement among raters in our pilot study [39]. Table 1 displays the items in their final wording.

Table 1: example items per construct

\begin{tabular}{|l|l|}
\hline $\begin{array}{l}\text { Theory } \\
\text { Construct }\end{array}$ & Item Definition \\
\hline
\end{tabular}




\begin{tabular}{|l|l|}
\hline EA practice & $\begin{array}{l}\text { What is the current status regarding the } \\
\text { Enterprise Architecture practice in your } \\
\text { organization? }\end{array}$ \\
\hline $\begin{array}{l}\text { Digital } \\
\text { transformation } \\
\text { journey }\end{array}$ & $\begin{array}{l}\text { Based on the information about } \\
\text { Dimensions and Levels from the previous } \\
\text { section, what is current status of the } \\
\text { Digital transformation within your } \\
\text { organization? }\end{array}$ \\
\hline $\begin{array}{l}\text { Resilience } \\
\text { Strategy }\end{array}$ & $\begin{array}{l}\text { Which of the following Resilience } \\
\text { strategies does your organization use to } \\
\text { handle disruptions? }\end{array}$ \\
\hline Strategy & $\begin{array}{l}\text { Which of the following Resilience } \\
\text { strategies do you think are the most } \\
\text { relevant for Enterprise Architecture? }\end{array}$ \\
\hline $\begin{array}{l}\text { EA Resilience } \\
\text { Strategy }\end{array}$ & $\begin{array}{l}\text { Which of the following Resilience } \\
\text { attributes do you think are the most } \\
\text { important to ensure a resilient Enterprise } \\
\text { Architecture? }\end{array}$ \\
\hline
\end{tabular}

\section{Findings and Conclusion}

By proposing a theory-led perspective on value generation through Enterprise Resilience, this study adds to the research stream on Enterprise Resilience benefit realization. Our findings have significant theoretical and practical implications. The instrument development procedure described in this paper provides several contributions. First, propose a way to integrate knowledge on several topics, namely, EA, IS, resilience, and digital transformation, under the umbrella of IT governance. Second, we reported on the process of developing a valid and reliable instrument to measure EA Resilience factors in context to digital transformation. We believe that this instrument can be used in various studies including our future research to examine the factors determining EA resilience in context to digital transformation in organizations. Third, we described in detail a model for instrument development. We believe that fellow researchers to be able to successfully adopt this model in their empirical studies.

Our study has some limitations, like any other research. First, there is limited research available on EA resilience, so we had to rely on knowledge from related fields, such as IS. While there is overlap between these two fields, we currently don't have good understanding of which aspects from IS resilience would also be applicable for EA resilience. Thus, we designed the questionnaire to collect opinions from EA practitioners on which aspects of IS resilience are also relevant for EA resilience. Second, the phenomenon we aim to study is very complex. While the conceptual model we designed focuses on the major concepts and relationships resulting from literature, we cannot ensure that it is complete as there could be additional concepts and relationships that are possible and relevant. Therefore, with this research, we hope to provide a starting point for further and more specific, studies on EA resilience.

Moreover, the framework for our analysis was IT governance and the RBV, which provided us with a rich set of concepts for the analysis of the EA value generation process. Despite the power of this approach, it might be fruitful to investigate how contextual factors, like culture, certain motivation mechanisms, and institutional processes, shape the building and exploitation of the EA Resilience capabilities. We plan on investigating these aspects in our future research using our instrument. In view of this study's contributions and limitations, we further propose that future research should use this study's findings and instrument that has been developed to measure changes in the identified EA Resilience capabilities and maturity. For instance, as suggested in our study, it would be valuable to learn how EA Resilience can help in building digital transformation capabilities in organizations embarking on a digital transformation journey. We also encourage further investigations of how frameworks, methods, and tools can support value generation in particular scenarios.

\section{References}

[1] A. Sarkar, S. C. Wingreen, and J. Ascroft, "Top management team decision priorities to drive IS resilience: Lessons from Jade Software Corporation," 2016.

[2] K. M. Eisenhardt and J. A. Martin, "Dynamic capabilities: what are they?," Strategic management journal, vol. 21, no. 10-11, pp. 1105-1121, 2000.

[3] D. E. Leidner, G. Pan, and S. L. Pan, "The role of IT in crisis response: Lessons from the SARS and Asian Tsunami disasters," The Journal of Strategic Information Systems, vol. 18, no. 2, pp. 80-99, 2009.

[4] A. Aldea, E. Vaicekauskaite, M. Daneva, and J. P. S. Piest, "Assessing Resilience in Enterprise Architecture: A Systematic Review," 2020 2020: IEEE, pp. 1-10.

[5] P. Weill and J. W. Ross, IT governance: How top performers manage IT decision rights for superior results. Harvard Business Press, 2004.

[6] O. Erol, D. Henry, B. Sauser, and M. Mansouri, "Perspectives on measuring enterprise resilience," 2010 2010: IEEE, pp. 587-592.

[7] A. Aldea, M. E. Iacob, J. van Hillegersberg, D. Quartel, and H. Franken, "Strategy on a Page: An ArchiMate-based tool for visualizing and designing strategy," Intelligent systems in accounting, finance and management, vol. 25, no. 2, pp. 86-102, 2018. 
[8] A. Aldea, M.-E. Iacob, D. Quartel, and H. Franken, "Strategic planning and enterprise achitecture," 2013 2013: IEEE, pp. 1-8.

[9] V. Gurbaxani and D. Dunkle, "Gearing Up For Successful Digital Transformation," MIS Quarterly Executive, vol. 18, no. 3, 2019.

[10] A. Priyono, A. Moin, and V. N. A. O. Putri, "Identifying Digital Transformation Paths in the Business Model of SMEs during the COVID-19 Pandemic," Journal of Open Innovation: Technology, Market, and Complexity, vol. 6, no. 4, p. 104, 2020.

[11] S. De Haes and W. Van Grembergen, "IT governance and its mechanisms," Information systems control journal, vol. 1, pp. 27-33, 2004.

[12] G. Müller, T. G. Koslowski, and R. Accorsi, "Resilience-a new research field in business information systems?," 2013 2013: Springer, pp. 3-14.

[13] C. S. Holling, "Resilience and stability of ecological systems," Annual review of ecology and systematics, vol. 4, no. 1, pp. 1-23, 1973.

[14] K. Burnard and R. Bhamra, "Organisational resilience: development of a conceptual framework for organisational responses," International Journal of Production Research, vol. 49, no. 18, pp. 5581-5599, 2011.

[15] D. Denyer, "Organizational Resilience: A summary of academic evidence, business insights and new thinking," BSI and Cranfield School of Management, pp. 8-25, 2017.

[16] S. Duchek, "Organizational resilience: a capabilitybased conceptualization," Business Research, vol. 13, no. 1, pp. 215-246, 2020.

[17] M. K. Linnenluecke, "Resilience in business and management research: A review of influential publications and a research agenda," International Journal of Management Reviews, vol. 19, no. 1, pp. 4-30, 2017.

[18] A. Sarkar, S. Wingreen, and J. Ascroft, "Towards a practice-based view of Information Systems Resilience Using the Lens of Critical Realism," 20202020.

[19] J. Ramezani and L. M. Camarinha-Matos, "Approaches for resilience and antifragility in collaborative business ecosystems," Technological Forecasting and Social Change, vol. 151, p. 119846, 2020.

[20] R. Bemthuis, M.-E. Iacob, and P. Havinga, "A Design of the Resilient Enterprise: A Reference Architecture for Emergent Behaviors Control," Sensors, vol. 20, no. 22, p. 6672, 2020.

[21] H. Lochhead, "Resilience by design: can innovative processes deliver more?," Procedia Engineering, vol. 180, pp. 7-15, 2017.

[22] I. Linkov, B. D. Trump, M. Golan, and J. M. Keisler, "Enhancing Resilience in Post-COVID Societies: By Design or By Intervention?," Environmental Science \& Technology, vol. 55, no. 8, pp. 4202-4204, 2021.

[23] F. Ahlemann, C. Legner, and J. Lux, "A resource-based perspective of value generation through enterprise architecture management," Information \& Management, vol. 58, no. 1, p. 103266, 2021.

[24] M. Meyer, M. Helfert, and C. O'Brien, "An analysis of enterprise architecture maturity frameworks," 2011: Springer, pp. 167-177.

[25] J. Vallerand, J. Lapalme, and A. Moïse, "Analysing enterprise architecture maturity models: a learning perspective," Enterprise Information Systems, vol. 11, no. 6, pp. 859-883, 2017.

[26] The Open Group, "The TOGAF® Standard, Version 9.2," The Open Group, 2018. [Online]. Available: https://pubs.opengroup.org/architecture/togaf9-doc/arch/

[27] M. Roelfsema, A. Aldea, M. Lankhorst, and H. Franken, "How about Strategy?: A Survey into the Pitfalls of Strategic Alignment," Journal of Enterprise Architecture, vol. 12, no. 1, pp. 7-18, 2016.

[28] T. Kaddoumi and M. Watfa, "A proposed agile enterprise architecture framework," 2016: IEEE, pp. 52-57.

[29] The Open Group, "The Open Group Agile Architecture Framework Draft Standard," The Open Group, 2021. [Online]. Available: $\quad$ https://pubs.opengroup.org/architecture/oaaf/snapshot/Agile Architecture Framework.html\#aafmaturity-model

[30] S. Schneider and O. Kokshagina, "Digital transformation: What we have learned (thus far) and what is next," Creativity and Innovation Management.

[31] R. Teichert, "Digital transformation maturity: A systematic review of literature," Acta universitatis agriculturae et silviculturae mendelianae brunensis, vol. 67, no. 6, pp. 1673-1687, 2019.

[32] B. Anthony Jnr, "Managing digital transformation of smart cities through enterprise architecture-a review and research agenda," Enterprise Information Systems, pp. 1-33, 2020 .

[33] O. Valdez-de-Leon, "A digital maturity model for telecommunications service providers," Technology innovation management review, vol. 6, no. 8, 2016.

[34] A. Rossmann, "Digital maturity: conceptualization and measurement model," in 39th International Conference in Information Systems, San Francisco, 2018.

[35] J. W. Creswell and V. L. P. Clark, Designing and conducting mixed methods research. Sage publications, 2017.

[36] B. Blumberg, D. Cooper, and P. Schindler, Business Research Methods. McGraw Hill, 2014.

[37] P. Lietz, "Research into questionnaire design: A summary of the literature," International journal of market research, vol. 52, no. 2, pp. 249-272, 2010.

[38] J. Recker, "Opportunities and constraints: the current struggle with BPMN," Business Process Management Journal, 2010.

[39] J. R. Landis and G. G. Koch, "The measurement of observer agreement for categorical data," biometrics, pp. 159174, 1977. 